\title{
Screening of Acute Kidney Injury in ICU Patients in Kafr-Elsheikh Governorate, Egypt
}

\author{
M.E.Ibrahim ${ }^{1}$, T.E.Haydara ${ }^{2}$, H.G.Abd Elsalam ${ }^{1}$, A.R.El.Sherbiny ${ }^{2}$ and A.E.Mansour ${ }^{1}$ \\ ${ }^{1}$ Internal Medicine Dept., Faculty of Medicine, Benha Univ., Benha, Egypt \\ ${ }^{2}$ Internal Medicine Dept., Faculty of Medicine, Kafr-Elsheikh Univ., Benha, Egypt \\ E-Mail:a.rha@gmail.com
}

\begin{abstract}
Intense kidney harm (AKI) is An significant worldwide wellbeing problem, which imposes An negative impact for tolerant horribleness What's more is answerable for an evaluated 1.4 million passings for every year. The point about this fill in might have been will figure out prevalence, causes, diagnosis, complications, necessity on dialysis alternately not, oversaw economy Furthermore prognosis for AKI clinched alongside Kafr Elsheikh Governorate doctor's facilities. Those consider incorporated 250 patients admitted in seriousness (ICU) On Kafr Elsheikh governorate doctor's facilities over six months. Patients were subjected for; full historical backdrop taking, physical examination Also research center examination Similarly as serum creatinine, pee Investigation for hematuria , pyuria , casts , cbc to anele , serum na , $\mathrm{k}$, CRP, RBS , liver capacity tests , ABG. Comes about Also conclusion: the examine included 250 patients, imply agdistis 49. $7 \pm$ 7. 2. Those contemplate incorporated 132(52. 8\%) male instances What's more 118(47. 2\%) female situations. Hypertension might have been introduce over $34 \%$ about patients, same time CHD might have been display for $12.8 \%$. Dm Furthermore Ceaseless liver infection were also available Anyhow during more level rates. Sepsis might have been the commonest precipitating variable to AKI clinched alongside these patients (61. 2\%) accompanied Eventually Tom's perusing drying out (53.6\%). Oliguria might have been An presentation manifestation in $45.6 \%$ about patients, irresistible side effects were exhibit to $45.6 \%$. Likewise view mortality, $(12.8 \%)$ from claiming patients passed on Throughout their confirmation. Finish renal recuperation might have been the The majority regular result from claiming AKI patients over our study $(53.2 \%)$.
\end{abstract}

Keywords: Acute kidney injury, AKI, ICU.

\section{Introduction}

AKI need been long distinguished Similarly as An normal muddling Previously, frigid patients. It need not been until that intensivist need demonstrated a developing enthusiasm toward and attention to its genuine sway once expenses Furthermore outcome, it may be acknowledged that AKI will be An as a relatable point clinical syndrome for frigid Furthermore need a focal part Likewise a systemic illness making different systemic sequels Also lesions over extra-renal organs. Also, AKI exerts An negative impact on the span What's more short- and long haul prognosis of the infection not main to those tolerant as well as to the kidney [1].

The principle reason for AKI are ischemia, hypoxia and nephrotoxicity. The instruments included over kidney damage and repair shed are mind boggling. The kidney is especially defenseless should ischemia Also toxins, bringing about vasoconstriction, endothelial damage, Furthermore actuation about incendiary procedures. This defenselessness arises To some degree from the vascular-tubular connections up the external medulla of the kidney, the place the halfway weight of oxygen is low, much at baseline, making them a greater amount powerless with An diminished renal blood stream. In the vicinity of a diminished glomerular filtration rate (GFR) optional will hypo perfusion, those ordinary reaction of the kidney is on maximally focus pee Furthermore reabsorb sodium avidly over an exert should maintain/increase intravascular volume What's more standardize renal perfusion. However, a prolonged diminishing to renal perfusion camwood bring about irreversible ischemic damage, prompting ischemic AKI alternately intense tubular corruption (ATN) [3].
Intense kidney damage (AKI) will be a clinical analysis guided Toward standard criteria In light of transforms Previously, serum creatinine, pee output, or both. Seriousness about AKI may be confirmed Eventually Tom's perusing those extent from claiming expansion for serum creatinine alternately diminishing for pee yield. Patients manifesting both oliguria and azotemia Furthermore the individuals done which these impairments are constant would less averse will have more awful infection Furthermore more awful results. Short- Furthermore long haul results are more awful At patients bring a portion stage of AKI by both criteria. New biomarkers to AKI might considerably help in the hazard appraisal Furthermore assessment about patients toward hazard for AKI [4]

For separate intense kidney harm renal supplanting treatment might be necessary [6].

The point for this worth of effort might have been with figure out prevalence, causes, diagnosis, complications, necessity on dialysis alternately not, management and prognosis of AKI done Kafr Elsheikh Governorate healing facilities.

\section{Patients and methods}

This cross-sectional observational study, might have been led for Kafr Elsheikh governorate doctor's facilities over six months with respect to 250 patients to frigid for typical kidney capacity tests at confirmation Also climbing kidney capacity tests Throughout frigid sit tight. Those examine might have been endorsed Toward morals panel for mankind's Scrutinize from claiming Benha employees for drug. 


\subsection{Inclusion criteria}

Age $>18$ years, frigid patients with ordinary kidney capacity tests toward confirmation Also climbing kidney capacity tests Throughout frigid remain. We utilized the late global kidney sickness moving forward worldwide results (KDIGOs) guideline [2].

\subsection{Exclusion criteria}

- known renal patients, CKD.

- renal transplant patients.

- Our Patients were subjected for;. full history bringing. - physical examination including pulse estimation systolic ,diastolic ,body weight, height, BMI, pulse, temperature. appraisal for volume status Toward observing CVP. cardiovascular examination to indications for heart disappointment. Abdominal examination for indications of incessant liver disappointment Concerning illustration Ascites, insect angiomas, caput medusa alternately indications from claiming parchedness Likewise diminished skin turgor What's more indications for vascular reason for inalienable renal disappointment Similarly as abdominal bruits/ midsection examination for indications about pulmonary clogging.

- lab examination serum creatinine assessed GFR utilizing CKD-EPI CYSTATIN c mathematical statement (2012).

\subsection{Statistical analysis}

Table (1) Relationship between laboratory criteria and prognosis in AKI studied patients.

\begin{tabular}{|c|c|c|c|c|}
\hline Factor & Complete recovery & Partial recovery & No recovery & Death \\
\hline $\begin{array}{l}\text { Serum Cr } \\
(\mathrm{mg} / \mathrm{dl})\end{array}$ & $2.2 \pm 0.5$ & $3.1 \pm 1.3$ & $4.2 \pm 1.7$ & $4.6 \pm 1.7$ \\
\hline $\begin{array}{l}\text { Serum urea } \\
(\mathrm{mg} / \mathrm{dl})\end{array}$ & $86.5 \pm 27.5$ & $132.7 \pm 72.0$ & $166.1 \pm 90.09$ & $192.97 \pm 87.7$ \\
\hline $\begin{array}{l}\text { GFR } \\
\left(\mathrm{ml} / \mathrm{min} \text { per } \mathrm{m}^{2}\right)\end{array}$ & $36.7 \pm 9.4$ & $28.8 \pm 12.27$ & $21.6 \pm 9.97$ & $18.1 \pm 11.07$ \\
\hline $\begin{array}{l}\text { Serum albumin } \\
\text { (g/dl) }\end{array}$ & $3.6 \pm 0.6$ & $3.4 \pm 0.8$ & $2.9 \pm 0.4$ & $2.0 \pm 0.2$ \\
\hline $\begin{array}{l}\text { Serum bilirubin } \\
(\mathrm{mg} / \mathrm{dl})\end{array}$ & $1.7 \pm 0.57$ & $2.2 \pm 0.7$ & $2.5 \pm 0.9$ & $5.0 \pm 4.67$ \\
\hline SGPT & $24 \pm 16.5$ & $25.2 \pm 19.09$ & $59.9 \pm 26.05$ & $538.61 \pm 436.89$ \\
\hline sGOT & $26.3 \pm 20.2$ & $36.6 \pm 23.6$ & $55.7 \pm 27.4$ & $467.2 \pm 351.69$ \\
\hline $\begin{array}{l}\text { Haemoglobin } \\
(\mathrm{g} / \mathrm{l})\end{array}$ & $9.8 \pm 1.4$ & $8.8 \pm 1.96$ & $8.5 \pm 1.4$ & $8.3 \pm 1.6$ \\
\hline Platelet count & $294357.1 \pm 110238.8$ & $265873.2 \pm 118772.2$ & $258481.4 \pm 137520.5$ & $224062.5 \pm 107981.5$ \\
\hline WBCs count & $11766.1 \pm 4543.9$ & $14421.4 \pm 5233.3$ & $14517.1 \pm 6451.2$ & $15562.5 \pm 4608.9$ \\
\hline $\mathrm{HCO}_{3}(\mathrm{mEq} / \mathrm{l})$ & $22.3 \pm 3.3$ & $18.4 \pm 3.5$ & $17.3 \pm 3.4$ & $11.9 \pm 6.1$ \\
\hline $\operatorname{Pco} 2(\mathrm{mEq} / \mathrm{l})$ & $32.8 \pm 6.5$ & $32.2 \pm 7.5$ & $31.3 \pm 6.8$ & $16.9 \pm 7.0$ \\
\hline PH & $7.4 \pm 0.1$ & $7.3 \pm 0.1$ & $7.2 \pm 0.1$ & $7.1 \pm 0.04$ \\
\hline INR & $1.2 \pm 0.2$ & $1.3 \pm 0.2$ & $1.3 \pm 0.3$ & $1.6 \pm 0.3$ \\
\hline $\begin{array}{l}\mathrm{C} \text { - } \\
\text { reactiveprotein }\end{array}$ & $16.1 \pm 10.2$ & $33.0 \pm 21.6$ & $40.6 \pm 4.6$ & $47.3 \pm 26.8$ \\
\hline $\mathrm{Na}^{+}(\mathrm{mEq} / \mathrm{l})$ & $131.0 \pm 6.2$ & $131.6 \pm 5.1$ & $127.7 \pm 4.4$ & $127.8 \pm 7.6$ \\
\hline $\mathrm{K}^{+}(\mathbf{m E q} / \mathbf{l})$ & $4.3 \pm 0.8$ & $4.4 \pm 0.6$ & $4.7 \pm 0.8$ & $5.0 \pm 0.5$ \\
\hline
\end{tabular}

Foray and dissection for information were performed by utilizing measurable one bundle to social Sciences (SPSS) adaptation 21. In this contemplate the qualitative variables were endorsed utilizing number Also percent, Chisquare test might have been utilized to examination (Mont Carlo correct test Also Fishers correct test were utilized as plan B to Chi-square test Assuming that there were a number little expected values). Numerical variables were communicated Similarly as intend \pm standard deviation, autonomous specimens t- test (for typical conveyed information) alternately Mann-Whitney U-test (for non-normal dispersed information) were utilized for correlation of methods qualities the middle of aggregations. Those event about demise might have been set Similarly as a indigent variable, Furthermore the sum variables noteworthy clinched alongside univariate examination were investigated Toward a numerous relapse logistic model, and the effects were tabulated Likewise chances degree What's more certainty interim. Pearson and Spearman relationship effective were used to survey correspondence between two constant variables. $\mathrm{P}$ worth $(\leq 0.05)$ might have been received Similarly as the level for hugeness.

\section{Results}

The study included 250 patients (mean age $49.7 \pm$ 7.2) admitted in ICU units in Kafr Elsheikh governorate hospitals over six months. The study included $132(52.8 \%)$ male cases and 118(47.2\%) female cases.

\footnotetext{
- Values are presented as mean \pm SD.
} 
Table (1) describes comparison of laboratory criteria of AKI studied patients in relation to prognosis. High serum creatinine, urea, liver enzymes, WBCs count, INR, CRP, low GFR and serum albumin were associated

with mortality. While low serum creatinine, urea, liver enzymes, WBCs count, INR, bilirubin, high GFR, s.albumin were associated with recovery with statistical significant difference. Complete renal recovery was observed when serum $\mathrm{Cr}$ value among AKI studied patients was $(2.2 \pm 0.5 \mathrm{mg} / \mathrm{dl})$.Partial renal recovery was observed when serum $\mathrm{Cr}$ value among AKI studied patients was $(3.1 \pm 1.3 \mathrm{mg} / \mathrm{dl})$.No renal recovery was observed when serum $\mathrm{Cr}$ value among AKI studied patients was $(4.2 \pm 1.7 \mathrm{mg} / \mathrm{dl})$. Death was observed when serum $\mathrm{Cr}$ value among AKI studied patients was $(4.6 \pm 1.7 \mathrm{mg} / \mathrm{dl})$.Complete renal recovery was observed when serum albumin among AKI studied patients was $(3.6 \pm 0.6 \mathrm{~g} / \mathrm{dl})$.Partial renal recovery was observed when serum albumin among AKI studied patients was (3.4 \pm 0.8 $\mathrm{g} / \mathrm{dl}$ ).No renal recovery was observed when serum albumin among AKI studied patients was $(2.9 \pm 0.4$ $\mathrm{g} / \mathrm{dl}$ ). Death was observed when serum albumin among AKI studied patients was $(2 \pm 0.2 \mathrm{~g} / \mathrm{dl})$.Complete renal recovery was observed when WBCs count among AKI studied patients was $(11766.1 \pm 4543.9)$.Partial renal recovery was observed when WBCs count among AKI studied patients was (14421.4 \pm 5233.3).No renal recovery was observed when WBCs count among AKI studied patients was(14517.1 \pm 6451.2).Death was observed when WBCs count among AKI studied patients was (15562.5 \pm 4608.9).Complete renal recovery was observed when $\mathrm{PH}$ value among AKI studied patients was $(7.4 \pm 0.1)$. No renal recovery was observed when $\mathrm{PH}$ value among AKI studied patients was(7.2 \pm 0.1 ).Death was observed when $\mathrm{PH}$ value among AKI studied patients was (7.1 \pm 0.04$)$.Complete renal recovery was observed when $\mathrm{HCO} 3$ value among AKI studied patients was $(22.3 \pm 3.3 \mathrm{mEQ} / \mathrm{l})$.Partial renal recovery was observed when $\mathrm{HCO} 3$ value among AKI studied patients was $(18.4 \pm 3.5 \mathrm{mEQ} / \mathrm{L})$.No renal recovery was observed when $\mathrm{HCO} 3$ value among AKI studied patients was $(17.3 \pm 3.4 \mathrm{mEQ} / \mathrm{l})$. Death was observed when $\mathrm{HCO} 3$ value among AKI studied patients was $(11.9 \pm 6.1 \mathrm{mEQ} / \mathrm{l})$.Complete renal recovery was observed when serum potassium level among AKI studied patients was $(4.3 \pm 0.8 \mathrm{mEQ} / \mathrm{l})$.Partial renal recovery was observed when serum potassium level among AKI studied patients was $(4.4 \pm 0.6 \mathrm{mEQ} / \mathrm{l})$.No renal recovery was observed when serum potassium level among AKI studied patients was $(4.7 \pm 0.8 \mathrm{mEQ} / \mathrm{l})$. Death was observed when serum potassium level among AKI studied patients was $(5.0 \pm 0.5 \mathrm{mEQ} / \mathrm{l})$.

Table (2) Description of laboratory results in relation to mortality of AKI patients.

\begin{tabular}{|c|c|c|c|c|}
\hline Factor & $\begin{array}{l}\text { Death } \\
(n=32)\end{array}$ & $\begin{array}{c}\text { Alive } \\
(n=218)\end{array}$ & T-test & P-value \\
\hline Serum Cr (mg/dl) & $4.80(2.10-8.60)$ & $2.30(1.50-8.0)$ & $\mathrm{U}=974.0^{*}$ & $<0.001^{*}$ \\
\hline Blood urea (mg/dl) & $189.0(47.0-389.1)$ & $89.0(45.0-358.5)$ & $\mathrm{U}=1265.0^{*}$ & $<0.001^{*}$ \\
\hline GFR $(\mathrm{ml} / \mathrm{min}$ per $\mathrm{m} 2)$ & $14.0(5.0-37.0)$ & $37.0(6.0-48.0)$ & $\mathrm{U}=1188.0^{*}$ & $<0.001^{*}$ \\
\hline Serum albumin (g/dl) & $1.9(1.7-2.7)$ & $3.7(2.0-5.0)$ & $\mathrm{U}=135.5^{*}$ & $<0.001^{*}$ \\
\hline Serum bilirubin (mg/dl) & $3.60(1.70-23.30)$ & $1.90(1.0-4.50)$ & $\mathrm{U}=678.5^{*}$ & $<0.001^{*}$ \\
\hline SGPT & $850.0(16.0-960.0)$ & $18.0(11.0-115.0)$ & $\mathrm{U}=1412.5^{*}$ & $<0.001^{*}$ \\
\hline sGOT & $652.2(27.0-800.0)$ & $20.0(11.40-135.0)$ & $\mathrm{U}=479.5^{*}$ & $<0.001^{*}$ \\
\hline Haemoglobin (g/l) & $8.3 \pm 1.6$ & $9.4 \pm 1.7$ & $\mathrm{t}=3.331^{*}$ & $0.001^{*}$ \\
\hline Platelet count & $\begin{array}{c}225000.0 \\
(74000.0-510000.0)\end{array}$ & $\begin{array}{c}277000.0 \\
(44000.0-510000.0)\end{array}$ & $\mathrm{U}=2393.5^{*}$ & $0.004^{*}$ \\
\hline WBCs count & $\begin{array}{c}16800.0 \\
(4500.0-20900.0)\end{array}$ & $\begin{array}{c}12050.0 \\
(2220.0-26000.0)\end{array}$ & $\mathrm{U}=2263.5^{*}$ & $0.001^{*}$ \\
\hline $\mathrm{HCO}_{3}(\mathrm{mEq} / \mathrm{l})$ & $11.9 \pm 6.1$ & $20.7 \pm 3.9$ & $\mathrm{t}=8.032^{*}$ & $<0.001^{*}$ \\
\hline Pco2 (mEq/l) & $16.9 \pm 7.0$ & $32.5 \pm 6.9$ & $t=11.941^{*}$ & $<0.001^{*}$ \\
\hline PH & $7.1 \pm 0.0$ & $7.3 \pm 0.1$ & $\mathrm{t}=23.154^{*}$ & $<0.001^{*}$ \\
\hline INR & $1.7(1.3-2.8)$ & $1.2(1.0-2.0)$ & $\mathrm{U}=664.0^{*}$ & $<0.001^{*}$ \\
\hline C-reactive protein & $59.0(8.0-93.0)$ & $17.2(4.0-91.0)$ & $\mathrm{U}^{* *}$ & $.000^{*}$ \\
\hline $\mathrm{Na}^{+}(\mathbf{m E q} / \mathbf{l})$ & $127.8 \pm 7.6$ & $131.0 \pm 5.8$ & $\mathrm{t}=2.263^{*}$ & $0.030^{*}$ \\
\hline $\mathrm{K}^{+}(\mathrm{mEq} / \mathbf{l})$ & $5.0 \pm 0.5$ & $4.4 \pm 0.7$ & $\mathrm{t}=5.907^{*}$ & $<0.001^{*}$ \\
\hline
\end{tabular}

- Values are expressed as mean \pm SD

** Mann-Whitney U -test. Values of its variables are expressed as median (minimum-maximum).

Data was expressed by median (minimum-maximum) if abnormally distributed quantitative variables

Data was expressed by mean \pm SD. if normally distributed quantitative variables

t: Student t-test $\quad$ U: Mann Whitney test $\quad \mathrm{p}$ : $\mathrm{p}$ value for comparing between the studied groups

*: Statistically significant at $\mathrm{p} \leq 0.05$

Table (2) shows comparison of laboratory results in relation to mortality of AKI patients. High serum creatinine, high serum urea, high serum bilirubin, high liver enzymes, leucocytosis, low GFR, low serum albumin and high serum $\mathrm{K}$ were associated with mortality with statistical significant difference. Death was observed when serum $\mathrm{Cr}$ among AKI studied patients was $4.80(2.10-8.60 \mathrm{mg} / \mathrm{dl})$, serum albumin was $1.9\left(1.7 \_2.7 \mathrm{~g} / \mathrm{dl}\right)$, WBCs count was 16800.0 (4500.0 20900.0), Platelet count was 225000.0 (74000.0 - 
510000.0), HCO3 value was $11.9 \pm 6.1 \mathrm{mEQ} / 1$, PH value $0.5 \mathrm{mEQ} / \mathrm{l})$ with statistical significant difference. was $7.1 \pm 0.0 \&$ serum potassium level was $5.0 \pm$

Table (3) Relationship between contributing factors and prognosis in AKI studied patients.

\begin{tabular}{|c|c|c|c|c|c|c|}
\hline Aetiology & Complete recovery & Partial recovery & No recovery & Death & $X^{2}$ & P-value \\
\hline $\begin{array}{l}\text { NSAIDs } \\
(n=75)\end{array}$ & $39(52.0 \%)$ & $19(25.3 \%)$ & $4(5.3 \%)$ & $13(17.3 \%)$ & 2.118 & 0.548 \\
\hline $\begin{array}{l}\text { Dehydration } \\
(n=134)\end{array}$ & $56(41.8 \%)$ & $50(37.3 \%)$ & $8(6.0 \%)$ & $20(14.9 \%)$ & $16.235^{*}$ & $0.001^{*}$ \\
\hline $\begin{array}{l}\text { ACEIs } \\
(n=114)\end{array}$ & $63(55.3 \%)$ & $33(28.9 \%)$ & $6(5.3 \%)$ & $12(10.5 \%)$ & 1.079 & 0.782 \\
\hline $\begin{array}{l}\text { Sepsis } \\
(n=153)\end{array}$ & $63(41.2 \%)$ & $60(39.2 \%)$ & $10(6.5 \%)$ & $20(13.1 \%)$ & $27.597^{*}$ & $<0.001^{*}$ \\
\hline $\begin{array}{l}\text { AGN } \\
(n=13)\end{array}$ & $5(38.5 \%)$ & $2(15.4 \%)$ & $2(15.4 \%)$ & $4(30.8 \%)$ & 6.784 & $\begin{array}{l}{ }^{\mathrm{MC}} \mathrm{p}= \\
0.064\end{array}$ \\
\hline $\begin{array}{l}\text { Obstruction } \\
(\mathbf{n}=25)\end{array}$ & $11(44.0 \%)$ & $13(52.0 \%)$ & $1(4.0 \%)$ & $0(0 \%)$ & $9.159^{*}$ & ${ }^{\mathrm{MC}} \mathrm{p} 0.018^{*}$ \\
\hline
\end{tabular}

* Significant when p value <0.05. * Mont Carlo exact test.* - Values are presented as number and percentage (\%). $\chi^{2}$ : Chi square test $\quad$ C: Monte Carlo $\quad \mathrm{p}$ : $\mathrm{p}$ value for comparing between the studied groups.

Table (3) shows comparison of contributing factors in relation to prognosis in AKI studied patients. (41.2\%)of patients with sepsis had complete recovery. $17.3 \%$ of NSAIDs taking patients died during their admission.5.3\% of ACEIs taking patients showed no recovery of renal function. percentage of NSAIDs taking patients developed compelete renal recovery (52.0\%) comparable to percentage of partial renal recovery (25.3\%).Significant percentage of dehydrated patients developed complete renal recovery $(41.8 \%)$ comparable to percentage of partial renal recovery \&death $(37.3 \% \& 14.9 \%)$ respectively. percentage of ACEIs taking patients developed complete renal recovery $(55.3 \%)$ comparable to percentage of partial renal recovery ,death \&no renal recovery $(28.9 \%, 10.5 \% \& 5.3 \%)$ respectively. Significant percentage of septic AKI patients developed complete renal recovery $(41.2 \%)$ comparable to percentage of partial renal recovery \&death $(39.2 \% \& 13.1 \%)$ respectively. Significant percentage of AKI studied patients with evidence of obstructive uropathy developed partial renal recovery $(52.0 \%)$ comparable to percentage of complete renal recovery $(44.0 \%)$.

Table (4) Description of contributing risk factors in relation to mortality of AKI patients: \% from raw.

\begin{tabular}{|c|c|c|c|c|c|}
\hline & & Death & Alive & $X^{2}$ & P-value \\
\hline DM & Present & $6(26.1 \%)$ & $17(73.9 \%)$ & \multirow{2}{*}{4.007} & ${ }^{\mathrm{FE}} \mathrm{p}=$ \\
\hline$(n=23)$ & Absent & $26(11.5 \%)$ & $201(88.5 \%)$ & & 0.092 \\
\hline HTN & Present & $12(14.1 \%)$ & $73(85.9 \%)$ & \multirow{2}{*}{0.200} & \multirow{2}{*}{0.654} \\
\hline$(n=85)$ & Absent & $20(12.1 \%)$ & $145(87.9 \%)$ & & \\
\hline CHD & Present & $2(6.3 \%)$ & $30(93.8 \%)$ & \multirow{2}{*}{1.411} & \multirow{2}{*}{0.393} \\
\hline$(n=32)$ & Absent & $30(13.8 \%)$ & $188(86.2 \%)$ & & \\
\hline CLD & Present & $2(18.2 \%)$ & $9(81.8 \%)$ & \multirow{2}{*}{0.299} & ${ }^{\mathrm{FE}} \mathrm{p}=$ \\
\hline$(n=11)$ & Absent & $30(12.6 \%)$ & $209(87.4 \%)$ & & 0.637 \\
\hline NSAIDs & Present & $13(17.3 \%)$ & $62(82.7 \%)$ & \multirow{2}{*}{1.973} & \multirow{2}{*}{0.160} \\
\hline$(n=75)$ & Absent & $19(10.9 \%)$ & $156(89.1 \%)$ & & \\
\hline ACEIs & Present & $12(10.5 \%)$ & $102(89.5 \%)$ & \multirow{2}{*}{0.971} & \multirow{2}{*}{0.325} \\
\hline$(n=114)$ & Absent & $20(14.7 \%)$ & $116(85.3 \%)$ & & \\
\hline Sepsis & Present & $20(13.1 \%)$ & $133(86.9 \%)$ & \multirow[b]{2}{*}{0.026} & \multirow{2}{*}{0.872} \\
\hline$(\mathbf{n = 1 5 3})$ Absent & & $12(12.4 \%)$ & $85(87.6 \%)$ & & \\
\hline Dehydration & Present & $20(14.9 \%)$ & $114(85.1 \%)$ & \multirow{2}{*}{1.169} & \multirow{2}{*}{0.280} \\
\hline$(n=134)$ & Absent & $12(10.3 \%)$ & $104(89.7 \%)$ & & \\
\hline AGN & Present & $4(30.8 \%)$ & $9(69.2 \%)$ & \multirow{2}{*}{3.967} & ${ }^{\mathrm{FE}} \mathrm{p}=$ \\
\hline$(n=13)$ & Absent & $28(11.8 \%)$ & $209(88.2 \%)$ & & 0.069 \\
\hline Obstruction & Present & $0(0.0 \%)$ & $25(100.0 \%)$ & & $\mathrm{FE} \mathrm{D}-0.053$ \\
\hline$(\mathbf{n}=\mathbf{2 5})$ Absent & & $32(14.2 \%)$ & $193(85.8 \%)$ & 4.077 & $\mathrm{p}=0.053$ \\
\hline
\end{tabular}

*Significant when $\mathrm{p}$ value $<0.05 . \quad * *$ Fishers exact test. -Values are presented as number $(\%)$.
$\chi 2$ : Chi square test
E: Fisher Exact
$\mathrm{p}$ : $\mathrm{p}$ value for comparing between the studied groups.

Table (4) shows that there is a statistical significant difference between presence of contributing risk factors and mortality of AKI patients, as DM, CLD, CHD, dehydration, sepsis, NSAIDs, ACEIs and obstruction.
Among contributing factors, (18.2\%) of CLD patients were associated with mortality. $26.1 \%$ and $6.3 \%$ of DM and CHD patients respectively were associated with mortality. Percentage of AKI studied patients with CLD 
\&DM had been died during their admission (18.2\%\&26.1\%)comparable to those with CHD,NSAIDs taking,ACEIstaking,HTN,sepsis\&dehydration $(6.3 \%, 17.3$ $\%, 10.5 \%, 14.1 \%, 13.1 \% \& 14.9 \%$ )respectively.Oliguria was a presentation symptom in $45.6 \%$ of patients, infectious symptoms were present in $45.6 \%$, while other clinical symptoms as dysuria, hypotension, haematuria, and coma were also present but at lower frequencies. Table (5).

Table (5) Description of clinical characteristics of AKI studied patients (Presenting symptoms).

\begin{tabular}{lc}
\hline Factor & Patient (n=250) \\
Presentation & \\
Oliguria & $114(45.6 \%)$ \\
Infection symptoms (fever, loin pain, rash, arthritis, & $114(45.6 \%)$ \\
gross hematuria and ulcers(oral or nasal) & $75(30 \%)$ \\
Dysuria & $75(30 \%)$ \\
Hypotension & $25(10 \%)$ \\
Haematuria & \\
\hline
\end{tabular}

As regard Treatment modalities, $(69.6 \%)$ of patients underwent conservative treatment alone and, $(8.8 \%)$, $(10 \%)$ and $(11.6 \%)$ needed haemodialysis once, twice and more than twice respectively. The most common therapeutic modality offered to patients in our study was conservative treatment $(69.6 \%)$. Once and twice
Hemodialysis sessions were offered by comparable percentages to patients in our study $(8.8 \%$ $\& 10 \%)$ respectively. Once Hemodialysis sessions was the least common therapeutic modality offered to AKI patients in our study $(8.8 \%)$.

Table (6) Description of clinical characteristics of AKI studied patients (therapeutic modalities).

\begin{tabular}{lc}
\hline Factor & Patient $(\mathbf{n}=\mathbf{2 5 0})$ \\
\hline Treatment & \\
Conservative & $174(69.6 \%)$ \\
Hemodialysis & \\
Once & $22(8.8 \%)$ \\
Twice & $25(10 \%)$ \\
More than twice & $29(11.6 \%)$ \\
\hline
\end{tabular}

Outcomes were all-cause patient mortality, dialytic need and renal recovery. As regard, renal recovery, complete improvement was observed in $(53.2 \%)$ of patients. Relative improvement was observed in $(28.4 \%)$ of patients and no recovery in $(5.6 \%)$ of patients. As regard mortality, $(12.8 \%)$ of patients died during their admission. Complete renal recovery was the most common outcome of AKI patients in our study (53.2\%).There was significant relation between percentage of Partial renal recovery \&patients who died during their admission (the former about double the latter) $(28.4 \% \& 12.8 \%)$ respectively. No renal recovery was the least common outcome of AKI patients in our study $(5.6 \%)$ Table (7).

Table (7) Description of clinical characteristics of AKI studied patients (outcome).

\begin{tabular}{lc}
\hline Factor & Patient $(\mathbf{n = 2 5 0})$ \\
\hline Outcome & \\
Complete recovery & $133(53.2 \%)$ \\
Partial recovery & $71(28.4 \%)$ \\
No recovery & $14(5.6 \%)$ \\
Death & $32(12.8 \%)$ \\
\hline
\end{tabular}

Table (8) show clinical outcomes in different groups of patients. percentage of AKI studied patients with no associated comorbidities showed complete renal recovery $(48.9 \%)$ comparable to those with $\mathrm{HTN}$,DM ,CHD, CLD\& multiple associated comorbidities $(29.3 \%, 3 \%, 9 \%, 4.5 \% \& 5.3 \%)$ respectively. percentage of AKI studied patients with DM \&HTN showed partial renal recovery $(7 \% \& 35.2 \%)$ comparable to those with no associated comorbidities, CHD , CLD\& multiple associated comorbidities $(36.6 \%, 15.5 \%, 4.2 \% \& 1.4 \%)$ respectively. percentage of AKI studied patients with HTN showed no renal recovery $(26.7 \%)$. percentage of AKI studied patients with CLD \&CHD had been died during their admission(6.3\%\&6.3\%) respectively comparable to those with multiple associated comorbidities, DM, HTN\& no associated comorbidities $(6.3 \%, 12.5 \%, 31.3 \% \& 37.5 \%)$ respectively.

Table (8) Description of prognosis in different groups of AKI patients. 


\begin{tabular}{lcccccccc}
\hline & $\begin{array}{c}\text { No associated } \\
\text { conditions } \\
(\mathbf{n = 1 1 0})\end{array}$ & $\begin{array}{c}\text { HTN } \\
(\mathbf{n = 7 8})\end{array}$ & $\mathbf{D M}$ & $\mathbf{C H D}$ & $\mathbf{C L D}$ & $\begin{array}{c}\text { Two or more } \\
\text { associated } \\
\text { conditions } \\
(\mathbf{n = 1 0})\end{array}$ & $\begin{array}{c}\boldsymbol{X}^{2} \\
(\mathbf{n = 2 8})\end{array}$ & $\begin{array}{c}\text { P- } \\
\text { Value }\end{array}$ \\
\hline $\begin{array}{l}\text { Complete } \\
\text { recovery }\end{array}$ & $65(48.9 \%)$ & $39(29.3 \%)$ & $4(3.0 \%)$ & $12(9.0 \%)$ & $6(4.5 \%)$ & $7(5.3 \%)$ & 6.726 & 0.238 \\
$\begin{array}{l}\text { Partial } \\
\text { recovery }\end{array}$ & $26(36.6 \%)$ & $25(35.2 \%)$ & $4(7.0 \%)$ & $11(15.5 \%)$ & $3(4.2 \%)$ & $1(1.4 \%)$ & 5.668 & 0.327 \\
$\begin{array}{l}\text { No } \\
\text { recovery }\end{array}$ & $7(46.7 \%)$ & $4(26.7 \%)$ & $1(6.7 \%)$ & $3(20 \%)$ & $0(0 \%)$ & $0(0 \%)$ & 2.015 & 0.797 \\
Death & $12(37.5 \%)$ & $10(31.3 \%)$ & $4(12.5 \%)$ & $2(6.3 \%)$ & $2(6.3 \%)$ & $2(6.3 \%)$ & 5.795 & 0.269 \\
\hline
\end{tabular}

\section{Discussion}

In our study, those average (IQR) agdistis for AKI patients might have been $49.7 \pm 7.2$ with extend (30-55) a considerable length of time. In turn study accounted a low recurrence from claiming AKI in patients over $60 \mathrm{~A}$ long time [35\%] [7]. In contradiction with this, in turn past ponder accounted higher rate of AKI patients over 60 A long time [65. 2\%] [8].

For our study, 47. $2 \%$ for patients were females, 52. $8 \%$ of patients were guys. This absence of sex Contrast might have been in understanding with that accounted Eventually Tom's perusing others the place guys were a greater amount incessant with have AKI [8].

For managing the study of disease transmission for AKI, it might be useful with recognizing both the helping alternately precipitating Components and in addition those copartnered comorbidities. Done our consider sepsis might have been discovered will make those commonest precipitating component to AKI patients $(61.2 \%)$, emulated by drying out $(53.6 \%)$, confirmation from claiming obstructive uropathy speak to $(10 \%)$ about know helping components should AKI. CLD and AGN were quell Eventually Tom's perusing tantamount rates Concerning illustration helping components with AKI clinched alongside our examine (4. $4 \% \& 5.2 \%$ ). NSAIDs Also HTN were spoken to by similar rates Similarly as helping components on AKI for our study ( $30 \% \& 34 \%)$ separately.

These discoveries would inagreement for the individuals of a past examine [9]. In understanding with the effects about our study, a few past investigations accounted that drying out and sepsis constitute those larger part of helping components to AKI [10]. A few creators recorded that sepsis might have been those initial helping component for AKI [11]. This could a chance to be attributable of the sort from claiming patients for these investigations who were critically sick frigid patients.

The pathogenesis from claiming renal torment over volume exhaustion Also sepsis will be great known.

Significant drying out prompts impeded renal perfusion for a resultant fall in glomerular slim filtration weight. In this setting, tubular work is commonly normal, renal reabsorption about sodium What's more water is increased, and thusly a concentrated pee will be shaped (urine osmolality $>500 \mathrm{mOsm} / \mathrm{kg}$ ). However, a denoted diminishment On renal perfusion might overpower auto regulation Furthermore precipitate a intense fall in GFR. This contrasts will AKI precipitated Eventually Tom's perusing spoiling. Extreme contamination contributes with AKI mostly by those sepsis-endotoxin-cytokine arbiter framework Also superimposed disseminated intravascular coagulation. In this condition, those hemodynamic for this kidney is impeded Indeed going without systemic hypotension [3].

Those dominant part for AKI patients On our examine required connected comorbidities Similarly as hypertension, DM, LCF alternately CHF, whichever Previously, seclusion or in consolidation. Dm , confirmation of obstructive uropathy and CHD were spoke to by similar rates Similarly as helping components with AKI Previously, our investigation (9. $2 \%, 10 \% \& 12.8 \%$ ) individually. NSAIDs What's more HTN were spoke to Toward similar rates Likewise helping components will AKI Previously, our examine (30\% \&34\%) individually.

These comes about are in understanding with formerly accounted for investigations Toward others [12]; they concentrated on frigid patients for AKI. In contradiction for this, over another past study, recently under A large portion of the patients $(45.4 \%)$ required restorative comorbidities Likewise $\mathrm{dm}$, hypertension What's more ischemic coronary illness [12].

To our study, the A large portion as a relatable point comorbidity might have been hypertension emulated by CHF, dm What's more CLD; $(34 \%, 12.8 \%, 9.2 \% \& 4$. $4 \%)$ individually. In understanding with this, past writing accounted for that hypertension might have been those practically incessant comorbidity connected with AKI taken after Toward CKD, DM, CHF Also CLD separately [13]. In spite of the fact that a few investigations for AKI bring plainly shown the vitality for Ceaseless premorbid states Likewise congestive heart failure, constant hypertension, diabetes mellitus and preexisting renal sickness in the improvemen for kidney disappointment [14], those correct instrument behind this companionship is not great recognized yet.

A standout amongst the demonstrations is that renal offended connected with these Ceaseless premorbid states might make attributed should impeded endothelium-derived vaso unwinding which may be often display for diabetes mellitus, hypertension, atherosclerosis, and heart failure, bringing about Confusing vasoconstriction alternately lessened vasodilatation for territorial hypoxia [15]. Other 
underlying elements to expanded hazard of AKI in patients for CKD incorporate confused autoregulation, abnormal vasodilatation, expanded affectability will diuretics Also nephrotoxic agents, age-related progressions for renal physiology Also copartnered heart disappointment which is as a relatable point clinched alongside CKD [16].

Those sway about research center information ahead mortal sin might have been likewise examined. Clinched alongside our study, AKI patients for leukocytosis, secondary serum creatinine, helter skelter serum urea, helter skelter serum bilirubin, helter skelter liver proteins needed a higher danger for mortal sin over different patients with Factual critical distinction. Secondary serum creatinine, secondary serum urea, helter skelter serum bilirubin, helter skelter liver enzymes, leukocytosis, low GFR, low serum egg whites Furthermore low serum k were connected with mortal sin with Factual critical Contrast. Demise might have been watched when serum cr "around AKI contemplated patients might have been $4.8\left(2.1 \_8.6 \mathrm{mg} / \mathrm{dl}\right)$, serum egg whites was1.9 (1.7_3. 3g/dl), WBCs check might have been (15562. 5 \pm 4608 . 8), Platelet check might have been (224062. 50 107981.4$)$, HCO3 quality might have been 8. 5(6. 0_19. 6mEQ/1), ph worth might have been 7. 2(7. 1_7. 4) \& serum potassium level might have been 4. 4(3. 0_6. $0 \mathrm{mEQ} / \mathrm{l}$ ) for measurable critical distinction.

This finding might be demonstrated Eventually Tom's perusing the certainty that leukocyte Also helter skelter liver proteins Also helter skelter serum creatinine would lab variables conceivably connected with sepsis which required been watched Similarly as a free predictor for mortal sin [17].

\section{Conclusion}

In this study, AKI might have been more basic over males, hypertension might have been exhibit to $34 \%$ of patients, same time CHD might have been display clinched alongside $12.8 \%$. Dm Furthermore constant liver infection were also introduce in any case In bring down rates. Sepsis might have been those commonest precipitating figure to AKI done these patients (61.2\%) trailed Toward drying out (53.6\%), NSAIDS (30\%), utilization of ACEI (45.6\%), AGN (10\%), Also urinary tract block (5. $2 \%$ ). Oliguria might have been a presentation manifestation for $45.6 \%$ of patients, irresistible indications were display over $45.6 \%$, same time different clinical side effects Similarly as dysuria, hypotension, haematuria, What's more unconsciousness were likewise display Anyhow toward easier frequencies. Concerning illustration respect mortality, (12. 8\%) about patients passed on Throughout their confirmation. Finish renal recuperation might have been those mossycup oak regular Conclusion of AKI patients to our contemplate $(53.2 \%)$.

\subsection{Author contribution}

Authors contributed equally in the study.

\subsection{Conflicts of interest}

No conflicts of interest.

\section{References}

[1] G.Seller-Pérez, S.Más-Font, C.Pérez-Calvo. Acute kidney injury: Renal disease in the ICU. Med Intensiva (English Ed.vo.40(6), PP.374-82,2016.

[2] M.E.Thomas, C.Blaine, A.Dawnay. The definition of acute kidney injury and its use in practice. Kidney Int, Vol.87(1), PP.62-73,2015.

[3] D.P.Basile, M.D.Anderson, T.A.Sutton. Pathophysiology of acute kidney injury. Compr Physiol, Vol.2(2), PP.1303-53,2011.

[4] J.A.Kellum, F.E.Sileanu, R.Murugan.Classifying AKI by urine output versus serum creatinine level. J Am Soc Nephrol, Vol.26(9), PP.2231-8,2015.

[5] R.Bellomo, S.Bagshaw, C.Langenberg. Pre-renal azotemia: a flawed paradigm in critically ill septic patients? In: Acute Kidney Injury. Karger Publishers, Vol.8, PP.1-9,2007.

[6] R.Wald, R.R.Quinn, J.Luo.Chronic dialysis and death among survivors of acute kidney injury requiring dialysis. Jama, Vol.302(11), PP.117985,2009 .

[7] M.E.Grams, M.M.Estrella, J.Coresh. National Heart and Blood Institute Acute Respiratory Distress Syndrome Network L. Fluid balance, diuretic use, and mortality in acute kidney injury. Clin J Am Soc Nephrol, Vol.6(5), PP.966-73,2011.

[8] E.M.Bucuvic, D.Ponce, A.L.Balbi. Risk factors for mortality in acute kidney injury. Rev da Assoc Médica Bras (English Ed, Vol.57(2), PP.15661,2011.

[9] R.L.Mehta, E.A.Burdmann, J.Cerdá, Recognition and management of acute kidney injury in the International Society of Nephrology 0by25 Global Snapshot: a multinational cross-sectional study. Lancet, Vol.387(10032), PP.2017-25,2016.

[10] L.Cole, R.Bellomo, W.Silvester, e Victorian Severe Acute Renal Failure Study Group JHR for th. A prospective, multicenter study of the epidemiology, management, and outcome of severe acute renal failure in a "closed" ICU system. Am J Respir Crit Care Med,Vol.162(1), PP.191-6,2000.

[11] S.M.Bagshaw, S.Uchino, R.Bellomo, Septic acute kidney injury in critically ill patients: clinical characteristics and outcomes. Clin J Am Soc Nephrol,Vol.2(3), PP.431-9,2007.

[12] E.S.Wijewickrama, G.M.Ratnayake, C.Wikramaratne, Incidences and clinical outcomes of acute kidney injury in ICU: a prospective observational study in Sri Lanka. BMC Res Notes,Vol.7(1), PP.305,2014.

[13] M.M.Schissler, S.Zaidi, H.Kumar, Characteristics and outcomes in community-acquired versus hospital-acquired acute kidney injury. Nephrology,Vol.18(3), PP.183-7,2013.

[14] M.D.Okusa, G.M.Chertow, D.Portilla. The nexus of acute kidney injury, chronic kidney disease, and World Kidney Day 2009. Am Soc Nephrol,Vol.3(1), PP.12-7,2009. 
[15] T.F.Lüscher. The endothelium as a target and mediator of cardiovascular disease. Eur J Clin Invest,Vol.23(11), PP.670-85,1993.

[16] M.Gürsu, S.Uzun, R.Kazancioğlu. Acute Kidney Injury and Chronic Kidney Disease: A Bidirectional
Road. Med Bull Haseki/Haseki Tip Bul,Vol.55(1), PP.22-32,2017.

[17] A.Melamed, F.J.Sorvillo. The burden of sepsisassociated mortality in the United States from 1999 to 2005: an analysis of multiple-cause-of-death data. Crit care,Vol.13(1), PP.28-33,2009. 